\title{
Electronic cigarettes: a systematic review of available studies on health risk assessment
}

\begin{abstract}
Objective: This paper primarily aimed to review articles which specifically quantified the risk of electronic cigarette's (e-cigarette) usage via the health risk assessment (HRA) approach. Methods: Systematic literature searches were conducted using PubMed search engine databases. Search terms such as "electronic cigarette", "e-cigarette", "electronic nicotine delivery systems", "electronic cigarette liquid", "electronic cigarette vapors", and "health risk assessment" were used to identify the relevant articles to be included in this review. To enable comparison, hazard quotient (HQ) and lifetime cancer risk (LCR) for the chemicals measured in the selected articles were calculated for three of the articles using the formula: [1] $\mathrm{HQ}=$ average daily dose (ADD)/reference dose (RfD) or exposure air concentration $(\mathrm{EC}) /$ reference concentration $(\mathrm{RfC})$; [2] LCR=lifetime average daily dose (LADD) $\times$ cancer slope factor $(\mathrm{CSF})$ or exposure air concentration $(\mathrm{EC}) \times$ inhalation unit risk (IUR). Results: Four articles pertaining to HRA of e-cigarettes were critically reviewed, three of the papers focused on specific chemicals namely nicotine, propylene glycol (PG), glycerol and 1,2-propanediol, while one article evaluated the health risks posed by heavy metals contained in e-cigarettes. The calculated HQs for the chemicals in this review had large variations. HQs of the six chemicals, i.e. nicotine, PG, glycerol, cadmium, ethylene glycol, nickel, aluminum and titanium, were found to have the potential to contribute to non-carcinogenic health risks. None of the LCR calculated had risks exceeding the acceptable limit. Conclusion: There are limited HRA studies and the ones that were available provided inconsistent scientific evidences on the health risk characterization arising from the usage of e-cigarettes. As such, there is a need to perform more studies on HRA of e-cigarettes by using uniformed and comprehensive steps and similar reference threshold levels of exposures.
\end{abstract}

Keyword: E-liquid; Electronic cigarettes (e-cigarettes); Hazard quotient; Health risk assessment (HRA); Margin of exposure; Risk characterization 